\title{
Bronchopulmonary dysplasia and postnatal growth in extremely premature black infants
}

\author{
Mary Ellen A. Bozynski ${ }^{a}$, Jeffrey M. Albert ${ }^{b}$, Ushanalini Vasan ${ }^{c}$, \\ Michael N. Nelson ${ }^{c}$, Linda K. Zak ${ }^{\mathrm{a}}$, Patricia M. Naughton ${ }^{\mathrm{c}}$ \\ 'University of Michigan Medical Center, Department of Pediatrics, Section of Newborn Services, Ann \\ Arbor, MI, 'University of Michigan, Department of Biostatistics, Ann Arbor, MI and 'Rush Presby- \\ terian St. Luke's Medical Center, Departments of Pediatrics and Psychology, Chicago, IL (U.S.A.)
}

Accepted for publication 31 August 1989

\section{Summary}

Bronchopulmonary dysplasia (BPD) may adversely affect the postnatal growth of the extremely premature infant; however, most studies have not controlled for birth weight. We studied 90 Black premature infants (mean birth weight $989 \pm 148$ g). Weight was recorded biweekly until discharge and at 4,8 , and 12 months of age corrected for prematurity. Infants with BPD $(N=23)$ were contrasted with infants without BPD $(N=67)$. Data were modeled using the Count model: Stage $I$ birth to term and Stage II term to 12 months. Birth weight was considered part of growth beginning in utero and multivariate analyses were used to control for BPD, gestational age, duration of hospitalization and socioeconomic status. After adjustment for birth weight, BPD did not explain the growth pattern. A lower gestational age was associated with a slower establishment of steady growth $(P<0.01)$, while an increased duration of hospitalization was associated with a lower growth rate $(P<$ 0.05). Growth in stage II was not explained by study variables. 'Catch-up' growth was seen in both infants with and without BPD. We conclude that differences in growth among infants with BPD are mainly attributable to birth weight. We speculate that poorer growth may be seen in a sub-group of infants with severe BPD.

bronchopulmonary dysplasia; growth; premature infant.

\section{Introduction}

The postnatal growth of extremely premature infants has been the subject of considerable research [1-5]. Data suggest long-term impact of some perinatal compli-

Correspondence to: Mary Ellen A. Bozynski, University of Michigan Medical Center, Department of Pediatrics, L3023 Women's Hospital/0254, Ann Arbor, MI 48109/0254, U.S.A. 
cations, especially bronchopulmonary dysplasia (BPD), on growth [6,7]. BPD is a chronic respiratory condition which results mainly from oxygen toxicity and barotrauma to the immature lung [8]. The incidence of BPD is increasing with the increased survival of extremely premature infants. Although infants with BPD usually have many other associated conditions, most studies of growth have not controlled for birth weight or other differences between infants with and without bronchopulmonary dysplasia. In addition, most studies have involved mixed racial populations and very few reports have focused solely on extremely premature infants.

This prospective study focused on the growth of extremely premature Black infants weighing $<1,200 \mathrm{~g}$ at birth and was designed to answer the following questions: (1) Are the patterns of weight gain different for infants with bronchopulmonary dysplasia as compared to those without bronchopulmonary dysplasia? (2) Do other factors, e.g. birth weight and gestational age, help explain the patterns? (3) Is there evidence during the first year of life that extremely premature infants 'catchup' to full term infants; i.e., achieve weights within two standard deviations of the mean for their corrected age $[9,10]$ ?

\section{Patients and methods}

\section{Patient population}

The subjects of this report include 90 premature Black infants with birth weights $<1,200 \mathrm{~g}$ admitted to the intensive care nursery between March 1979 and June 1983 who survived and were discharged home. Only infants whose growth was appropriate for gestational age were included in the study [11]. Weight was measured at birth to the nearest $10 \mathrm{~g}$ (Toledo scale, Toledo, $\mathrm{OH}$ ). Weights were recorded at birth and at every even numbered week until discharge, which usually occurred at postconceptional age 38-42 weeks, corrected for prematurity. Weights were then measured and recorded during each follow up visit at 4, 8, and 12 months age corrected for prematurity. BPD was defined using the criteria of Bancalari et al. [12] and socioeconomic status (SES) was assessed using the Hollingshead Four Factor Index [13], with classes $1-3$ ('high') contrasted with classes 4-5 ('low') SES (Table I).

While hospitalized, the infants were generally managed by critical care nurse practitioners using nutritional protocols aimed at the provision of 100-120 kilocal/ $\mathrm{kg}$ /day either parenterally or enterally as dictated by the infant's condition.

\section{Modeling of the growth data}

The growth data were modeled using the Count model [14]. This model, which is widely used in the growth literature, is robust with respect to missing values, and has been used to describe the growth pattern of premature infants $[15,16]$.

The growth of premature infants is characterized by a loss of weight over the first few days after birth, recovery to birth weight, and finally establishment of a linear increase. The model fits this pattern well and is generally expressed as:

$$
\text { weight }=B_{0}+B_{\mathrm{i}}(\text { time })+B_{2} \ln (\text { time }+1)+E
$$


where $B_{0}, B_{1}$ and $B_{2}$ are the regression parameters and $E$ is the random error. It follows from this model that velocity or growth rate is given (as a function of time) as $B_{1}+B_{2} /($ time +1$)$ and acceleration, as $-B_{2} /($ time +1$) . B_{2}$ can be interpreted as an indicator of the curvature in the curve specified by the Count model, or more precisely as the initial (time 0 ) deceleration or rate of weight loss after birth. In a plot of weight versus time, more pronounced curvature corresponds to larger absolute values of $B_{2}$. As time increases, acceleration tends to zero, and velocity tends to stabilize at value $B_{1} . B_{0}$ can simply be thought of as the expected weight at time zero.

The model was fitted in two stages. The first stage was from birth to term (40 weeks corrected age) or discharge if this occurred prior to 40 weeks; the second stage from 40 weeks to 12 months corrected age. Thus, for each individual, the full range of data points was fitted in two segments. The two curves were connected using the predicted week 40 weight from the first model as the intercept for the second model. This procedure reflects the relative importance attributed to the first stage model. Our models were therefore:

\section{Stage I}

Expected wt gain at age $j=B_{0}+B_{1}($ age $j)+B_{2} \ln ($ age $j+1)$

\section{Stage II}

$$
\text { Expected additional wt at time } k=B_{3}(\text { time } k)+B_{4} \ln (\text { time } k+1)
$$

For stage I, age was postnatal age in weeks and for stage II, time $k$ was weeks from term corrected age. Note that for stage I the first observation was considered to be at birth. In doing so, birth weight was considered not as a covariate but as part of the overall growth already occurring in utero prior to birth [17].

After fitting the two stage model to each infant's data, a second phase in the analysis examined the influence of potential independent variables on the fitted curves. Variables examined included BPD, gestational age, duration of hospitalization, sex, and socioeconomic status. To do this, the estimated parameters for each individual were considered as a set of possibly-correlated random variables, i.e. as a multivariate observation. Using multivariate models it was possible to test whether a given covariate or set of covariates explained the set of estimates $b_{0}, \ldots b_{4}$, (the least squares estimates of $B_{0}, \ldots B_{4}$ ). All statistical computations were performed using SAS (SAS Institute Inc., Cary, NC)

After the corrected age of 40 weeks, the growth of study infants was plotted on the Babson curve for preterm infants [18] and compared to a population of Black full-term infants who were followed in an affiliated health maintenance organization at the same medical center [19].

Of the study infants, 64 contributed at least three data points to the modeling of stage II. The percent follow-up at each time point was: 77.5 (4 months), 68.5 (8 months), and 64.0 (12 months). 


\section{Results}

\section{Stage I Model}

The birth weights of $82.2 \%$ of the study infants fell between the 10th and 50th percentiles and the remaining $17.8 \%$ were between the 50 and 75 th percentiles for gestational age [11]. Sample characteristics are given in Table I. Perinatal variables for which infants with and without BPD differed are given in Table II.

For the first stage model, values for $b_{0}, b_{1}$, and $b_{2}$ were obtained for each infant and due to their interpretations are referred to as birth weight, growth rate, and deceleration, respectively. One infant had insufficient data on which to fit the model, i.e. less than three measurements. As expected, the three parameter estimates were highly correlated $(P<0.001)$; birth weight and growth rate were positively correlated, while birth weight was negatively correlated with deceleration, as were deceleration and growth rate.

Multivariate test statistics were utilized to control for correlation among variables. The results of the multivariate tests of the simultaneous effects of each covariate on the three parameter estimates are given in Table III. We also examined each of the covariates; BPD, gestational age and weeks hospitalization for their effects on growth rate and deceleration while controlling for the effect of birth weight (Table IV). The variable, weeks hospitalization, was highly correlated with both days of oxygen therapy $(r=0.54, P \leqslant 0.001)$ and days of mechanical ventilation $(r=0.66, P \leqslant 0.001)$, and thus served as a marker of more severe BPD.

\section{Bronchopulmonary dysplasia}

The first set of tests showed BPD closely linked to birth weight, growth rate and deceleration (Table III). The plots of fitted curves for infants with and without BPD are shown in Fig. 1. When adjustment was made for birth weight, however, the effect of BPD was no longer significant (Table IV). Thus, BPD was related to birth weight but did not further explain the subsequent pattern of growth.

\section{Gestational age}

The effect of gestational age is given in Table III. When adjusted for birth weight, lower gestational age was related to greater rate of weight loss or a slower establishment of a steady growth rate (Table IV). The eventual growth rate appeared lower for the earlier babies (Table III); however, when birth weight was taken into

\section{TABLE I}

Sample characteristics $(N=90)$.

\begin{tabular}{lc}
\hline Characteristic & Finding \\
\hline Mean birth weight, g (S.D.) & $989(148)$ \\
Mean gestational age, weeks (S.D.) & $28.5(1.9)$ \\
Mean hospitalization, weeks (S.D.) & $10.8(3.9)$ \\
Bronchopulmonary dysplasia, number (\%) & $23(25.6)$ \\
Socioeconomic status (class 4-5), number (\%) & $73(85.9)$ \\
Males number (\%) & $43(48.3)$
\end{tabular}




\section{TABLE II}

Perinatal variables for which risk groups differed. All comparisons were significant with $P<0.001$.

\begin{tabular}{llc}
\hline Perinatal variable & \multicolumn{2}{l}{ Risk group } \\
\cline { 2 - 3 } & No BPD & BPD \\
& $(N=67)$ & $(N=23)$ \\
\hline Mean birth weight-mean, (S.D.) & $1031(121)$ & $866(153)$ \\
Mean gestational age, weeks (S.D.) & $28.9(1.7)$ & $27.2(1.9)$ \\
Mean hospitalization, weeks (S.D.) & $9.5(3.0)$ & $14.3(3.9)$ \\
\hline
\end{tabular}

account (Table IV), there was no longer a significant effect of gestational age on eventual growth rate. In this sense, an earlier born infant will achieve the same growth rate as a later born infant if the two infants have similar birth weights.

\section{Weeks hospitalized}

Although weeks hospitalized alone was significantly related to birth weight and growth rate (Table III), when adjusted for birth weight, weeks hospitalization was no longer significantly related to growth rate (Table IV). On the other hand, the multivariate test, which took into account the correlation between growth rate and deceleration, indicated that weeks hospitalization significantly affected the two variables as a pair $(P<0.001)$. The two variable model with gestational age and weeks hospitalized (adjusted for birth weight) explained $33.0 \%$ of the variance for both growth rate and deceleration (Table V).

Since weeks hospitalization was highly correlated with both duration of mechanical ventilation and oxygen therapy, we substituted these variables for weeks hospitalization in the model. Similar results were obtained with duration of mechanical ventilation, explaining $35.3 \%$ of the variance in growth rate and $35.5 \%$ of the variance in deceleration. Days oxygen therapy was also significant but explained less of the variance in growth rate and deceleration as compared to both weeks hospitalization and duration of mechanical ventilation.

\section{TABLE III}

Tests and parameter estimates of multivariate regression models each involving one explanatory variable.

\begin{tabular}{llllll}
\hline Covariate & \multicolumn{2}{l}{$\begin{array}{l}\text { Test of covariate } \\
\text { effects }\end{array}$} & \multicolumn{5}{l}{$\begin{array}{l}\text { Estimate of covariate effects } \\
\text { (\%) variance) explained }\end{array}$} \\
\hline BPD & $F$-stat & $\begin{array}{l}P \text {-value } \\
\text { Weeks }\end{array}$ & BW & Growth rate & Deceleration \\
hospitalization & 11.5 & 0.0001 & $-162.7^{* *}$ & $-53.6^{* *}(16.1)$ & $86.2^{*}(4.7)$ \\
GA & 21.0 & 0.0001 & $-18.5^{* *}$ & $-6.4^{* *}(17.2)$ & $2.0(0.2)$ \\
SES & 42.7 & 0.001 & $42.3^{* *}$ & $8.8^{* *}(7.9)$ & $10.4(1.3)$ \\
& 1.5 & 0.22 & & & \\
\hline
\end{tabular}

GA, gestational age; SES, socio-economic status.

${ }^{*} P \leqslant 0.05$.

$* * P \leqslant 0.01$. 
TABLE IV

Tests and parameter estimates of multivariate regression models explaining growth rate and deceleration.

\begin{tabular}{|c|c|c|c|c|}
\hline \multirow[t]{2}{*}{ Covariate } & \multicolumn{2}{|c|}{$\begin{array}{l}\text { Test of covariate } \\
\text { effects }^{\mathrm{a}}\end{array}$} & \multicolumn{2}{|c|}{$\begin{array}{l}\text { Estimate of } \\
\text { covariate effects ( } \% \text { variance) }\end{array}$} \\
\hline & $F$-stat & $P$-value & Growth rate & Deceleration \\
\hline BPD & 2.2 & 0.12 & & \\
\hline GA & 22.4 & 0.0001 & $-2.76(0.6)$ & $52.3^{*}(22.3)$ \\
\hline $\begin{array}{l}\text { Weeks } \\
\text { hospitalization }\end{array}$ & 13.2 & 0.0001 & $-3.05(4.8)$ & $-8.5(2.6)$ \\
\hline
\end{tabular}

Adjusted for birth weight.

$* P \leqslant 0.01$.

\section{Conclusion}

\section{Stage I}

The pattern of growth from birth to term (corrected age) was best explained by birth weight, gestational age, and duration of hospitalization. BPD was related to birth weight but did not further explain the pattern of growth. A lower gestational age was associated with a slower achievement of steady weight gain, while an increased duration of hospitalization was associated with a lower growth rate. Neither sex nor socioeconomic status affected the growth pattern.

\section{Stage II}

Since the stage II parameters were derived from a second Count model connected

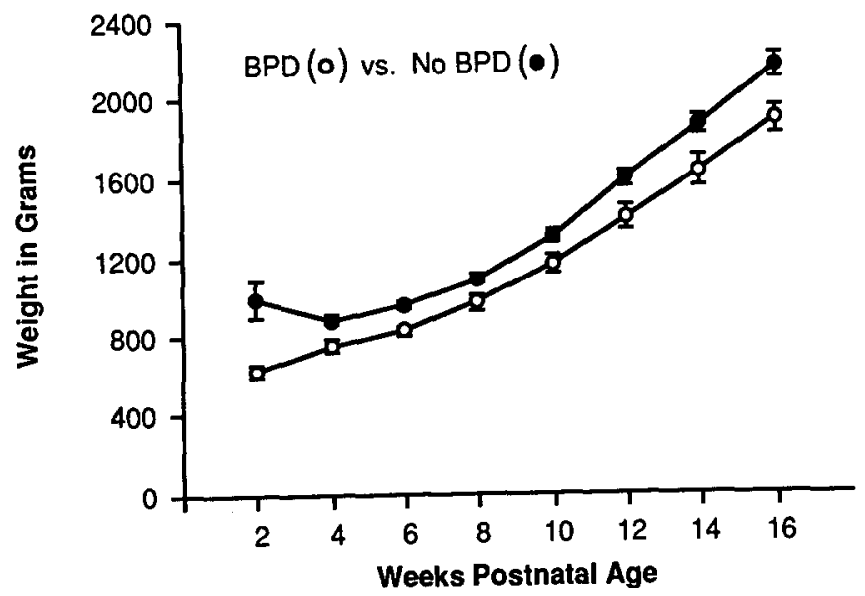

Fig. 1. Fitted curves for infants with and without BPD : birth to term corrected age. 


\section{TABLE V}

Tests and parameter estimates of the best multivariate regression model.

\begin{tabular}{llllll}
\hline $\begin{array}{l}\text { Two covariate } \\
\text { model }\end{array}$ & \multicolumn{2}{l}{$\begin{array}{l}\text { Test of corvariate } \\
\text { effects" }\end{array}$} & $\begin{array}{llll}\text { Estimate of } \\
\text { covariate effects }\end{array}$ & \\
\cline { 2 - 3 } \cline { 5 - 6 } & $F$-stat & $P$-value & & 'Growthrate' & 'Deceleration' \\
\hline GA' & 16.6 & 0.0001 & & -5.38 & $51.2^{* *}$ \\
Weeks & 8.2 & 0.0001 & & $-3.78^{*}$ & -1.6 \\
hospitalization & & & & \\
\hline
\end{tabular}

"Adjusted for birthweight.

$* P<0.05$.

$* * P<0.01$.

to the first model, there were only two parameters in the model which indicated growth rate and acceleration. A similar analysis to that performed for the first stage failed to show any significant covariate effects on the growth pattern. When infants who dropped out were compared to those who remained in the study, there were no differences for duration of oxygen or ventilator therapy. Those infants who dropped out were more likely to have exhibited poorer growth at the preceeding visit; however, the proportion of such infants did not differ for BPD and non-BPD groups.

\section{'Catch-Up'}

Most infants 'caught-up' or achieved weights within two standard deviations of the mean for their corrected age over time. At 4 months corrected age, $65 \%$ of infants with BPD had weights more than 2 S.D. below the mean compared to $40 \%$ of infants without BPD ( $P<0.001$, Fisher's exact test, two-sided). At 8 and 12 months, however, there was no difference between the groups. Growth of study infants in stage II, especially those without BPD, was fairly stereotyped and approximately 1 S.D. below the mean when plotted on the Babson chart or contrasted with a full-term comparison group (Fig. 2) [18,19].

\section{Discussion}

In this study of extremely premature Black infants of lower socioeconomic status, the differences in growth patterns from birth to term for infants with BPD as compared to infants without BPD were mainly attributable to the difference in birth weight in the two groups. When birth weight was taken into account, BPD did not appear to confer an added risk for poorer weight gain. On the other hand, gestational age and weeks hospitalization had a significant impact on the pattern of growth from birth to term. A lower gestational age was associated with a slower achievement of steady weight gain, while an increased duration of hospitalization was associated with a lower growth rate.

Duration of hospitalization may function as a marker for the severity of BPD. In 


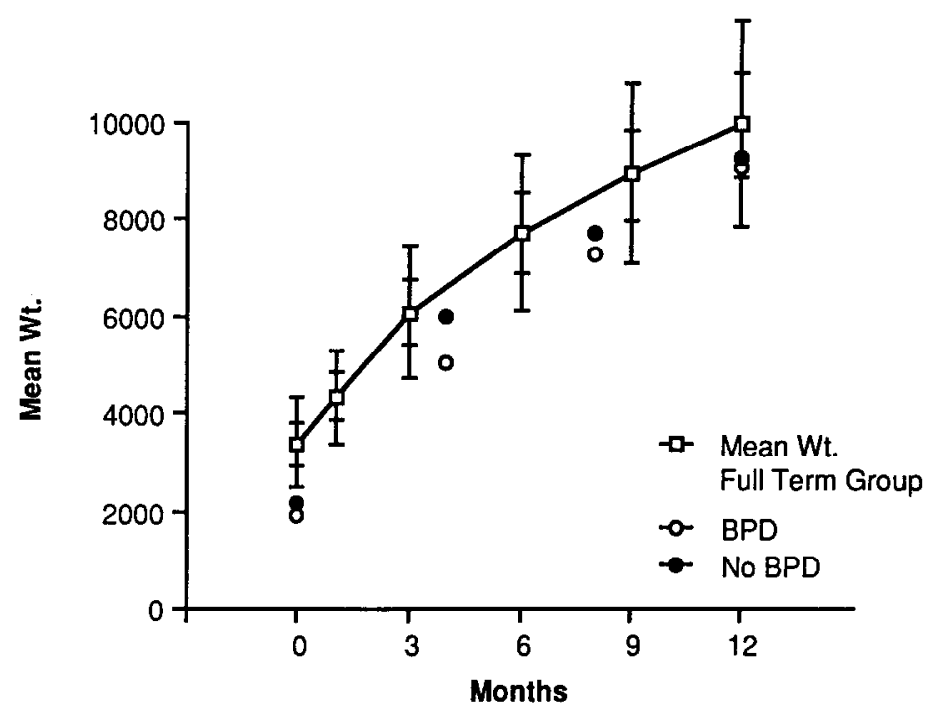

Fig. 2. Plot of fitted curves for infants with and without BPD and mean weights ( \pm 1 and 2 S.D.) for comparison infants during stage 2 - term to 12 months corrected age. Data for comparison infants adapted from Jung et al [19].

our study, the duration of hospitalization, days of oxygen therapy, and days of mechanical ventilation were all highly correlated. Furthermore, when duration of mechanical ventilation was substituted for weeks hospitalization, almost identical results were obtained. Perhaps further studies in which the severity of BPD is more precisely assessed will show a clearer impact of severe BPD on weight gain, even when other variables such as gestational age and birth weight are considered. This would not be surprising since a number of studies have shown increased work of breathing and increased oxygen consumption in infants with BPD [20,21].

We are unaware of any other studies of the impact of BPD on extremely premature Black infants of lower socioeconomic status. Although most studies of mixed populations have not included control groups of similar infants without BPD, several studies which have attempted to control for these factors have reached different conclusions. Vohr et al. found no difference in growth between BPD and control infants at 2 years of age [5]. On the other hand, Meisels et al. found poorer growth in infants with BPD as compared to infants with respiratory distress syndrome and no BPD in the second year of life [6]. Suave et al. examined the growth of infants with BPD as compared to controls and also found evidence for poorer growth in the infants with BPD [8]. In both of the two preceding studies, however, infants with BPD had lower birth weights. As seen in our study and from data recently published by Sell and Vaucher [22], the pattern of growth for BPD infants does not appear significantly different from that of similar infants when birth weight is taken into account. Sell and Vaucher found that body weight prior to 8 months of age explained $70-85 \%$ of the variance in subsequent weight [22]. 
Recent data published by Kurzner et al. suggest that both birth weight and severity of BPD are important [21]. Thirteen infants with BPD were studied at 6 months (corrected age). None were receiving supplmental oxygen. Seven had growth failure defined as weight less than tenth percentile of the Babson growth curve. Resting metabolic expenditure was increased among infants with both BPD and growth failure as compared to typically growing infants with or without BPD. The authors speculated that the growth failure was related to increased metabolic demands associated with BPD. Although birth weights of infants with growth failure were significantly lower $(P<0.005)$ than those of infants without growth failure, no adjustment for birth weight was made. Further studies which control for other factors, including clinical practices (e.g., diuretics, fluid restriction) are necessary. In addition, outcome variables other than weight gain alone, i.e., length and body composition, should be measured.

Among infants with or without BPD, there is a tendency for fewer premature infants to fall below the tenth percentile over time. Whether this trend toward fewer infants falling below the tenth percentile represents 'catch-up' growth of the premature infant to the normal full term population or simply 'catch-up' to growth predicted by their birth size is a matter for further consideration and study. Garn and Shaw [23] have pointed out that for normal term infants, birth size is the most important single determinant of individual growth during the first 7 years of life. Others have linked low birth weight and the rate of intrauterine growth to later growth [17]. In a recent study, Binken et al. plotted growth curves for low birth weight infants by 500-g weight groups [24]. The growth curves were highly divergent during early infancy, but began to converge toward the end of the first year. The curves never converged, however, and became parallel throughout early childhood, suggesting a persistent effect of birth weight. Both our study and the literature support the need for further research to clearly define the relative contribution of BPD and birth weight to postnatal growth patterns of the extremely premature infant.

\section{References}

1 Dancis, J., O'Connell, J.R., and Holt, L.E. (1948): A grid for recording the weight of premature infants. J. Pediatr., 33, 570-572.

2 Brandt, I. (1985): Growth dynamics of low-birth-weight infants. Acta Paediatr. Scand., Suppl., 319, 38-47.

3 Forslund, M. and Bjerre, I. (1985): Growth and development in preterm infants during the first 18 months. Early Hum. Dev., 10, 201-216.

4 Hack, M., Merkatz, I.R., Gordon, D., Jones, P.K., Fanaroff, A.A. (1982): Catch-up growth in very-low-birth-weight infants. Am. J. Obstet. Gynecol., 143, 693-699.

5 Vohr, B.R., Bell, E.F. and Oh, W. (1982): Infants with bronchopulmonary dysplasia. Growth pattern and neurologic developmental outcome. Am. J. Dis. Child., 136, 443-447.

6 Meisels, S.J., Plunkett, J.W., Roloff, D.W., Pasick, P.L. and Stiefel, G.S. (1986): Growth and development of preterm infants with respiratory distress syndrome and bronchopulmonary dysplasia. Pediatrics, 77, 345-352.

7 Sauve, R.S. and Singhal, N. (1985): Long-term morbidity of infants with bronchopulmonary dysplasia. Pediatrics, 76, 725-733. 
8 Platzker, A.C.G. (1988): Chronic lung disease of infancy. In: Pediatric Care of the ICN Graduate, pp.129-156. Editor: R.A. Ballard, W.B. Saunders, Philadelphia.

9 Hack, M., Merkatz, I.R., Mc Grath, S.K., Jones, P.K. and Fanaroff, A.A. (1984): Catch-up growth in very-low-birth-weight infants. Am. J. Dis. Child., 138, 370-375.

10 Karniski, W., Blair, C. and Vitucci, J.S. (1987): The illusion of catch-up growth in premature infants. Am. J. Dis. Child., 141, 520-526.

11 Lubchenko, L., Hansman, C. and Boyd, E. (1966): Intrauterine growth in length and head circumference as estimated from live births at gestational ages from 26 to 42 weeks. Pediatrics, 37, 403408.

12 Bancalari, E., Abdenour, G.E., Feller, R. and Gannon, J (1979): Bronchopulmonary dysplasia: clinical presentation. J. Pediatr., 95, 819-823.

13 Hollingshead, A. (1975): Four-factor index of social status. Yale Station, New Haven, CT.

14 Count, E.W. (1943): Growth patterns of human physique: An approach to kinetic anthropometry. Hum. Biol., 15, 1-32.

15 Smith, E.O., Schanler, R.J., Garza, C. and Nichols, B.L. (1983): Modeling the growth pattern of premature infants. Growth, 47, 340-347.

16 Berkey, C.S. (1982): Comparison of two longitudinal growth models for preschool children. Biometrics, 38, $221-234$.

17 Beck, G.J. and van den Berg, B.J. (1975): The relationship of the rate of intrauterine growth of low birthweight infants to later growth. J. Pediatr, 86, 504-511.

18 Babson, S.G. and Benda, G.I. (1976) Growth graphs for the clinical assessment of infants of varying gestational age. J Pediatr., 89, 814-820.

19 Jung, E. and Narins-Czajka, D. (1986) Comparison of growth of Black and White infants during their first two years of life. Natl. Med. Assoc., 78, 1157-1160

20 Weinstein, M.R. and Oh, W. (1981) Oxygen consumption in infants with bronchopulmonary dysplasia. J. Pediatr., 99, 958-961.

21 Kurzner S.I., Garg M., Bautista D.B., Bader D., Merritt, R.J., Warburton, D. and Keens, T.G. (1988) Growth failure in infants with bronchopulmonary dysplasia: nutrition and elevated resting metabolic expenditure. Pediatrics, 81, 379-384.

22 Sell, E.J. and Vaucher, Y. (1988) Growth and developmental outcome of infants who had bronchopulmonary dysplasia. In: Bronchopulmonary Dysplasia, pp. 403-420. Editors: T.A. Merritt, W.H. Northway, and B.R. Boynton. Blackwell Scientific Publications, Boston.

23 Garn, S.M. and Shaw, S.A. (1977): Birth size and growth appraisal. J. Pediatr., 90, 1049-1051.

24 Binkin, N.J., Yip, R., Fleshood, L. and Trowbridge, F.L. (1988): Birth weight and childhood growth. Pediatrics, 82, 828-834. 